\title{
Bipolar hip arthroplasty in geriatric patients using a conjoined tendon-preserving posterior approach
}

Tomonori Tetsunaga ( $\nabla$ tomonori_t31@yahoo.co.jp )

Okayama University https://orcid.org/0000-0003-4348-9806

\section{Tomoko Tetsunaga}

Okayama University Hospital

Kazuki Yamada

Okayama University

\section{Tomoaki Sanki}

Okayama University

Yoshi Kawamura

Okayama University

Toshifumi Ozaki

Okayama University

\section{Research article}

Keywords: Bipolar hip arthroplasty; geriatric patients; a conjoined tendon-preserving posterior approach

Posted Date: May 4th, 2020

DOl: https://doi.org/10.21203/rs.3.rs-25407/v1

License: (c) (i) This work is licensed under a Creative Commons Attribution 4.0 International License.

Read Full License 


\section{Abstract \\ Background}

In bipolar hemiarthroplasty (BHA), it is important to preserve soft tissue to reduce the risk of postoperative dislocation. While a variety of surgical approaches have been reported recently, extra care is needed with muscle- and tendon-preserving approaches in geriatric patients due to the fragility of their soft tissue. We investigated the usefulness of BHA using a conjoined tendon-preserving posterior (CPP) approach, which only dissects the external obturator muscle, in geriatric patients.

\section{Methods}

This retrospective study included 40 femoral neck fracture patients ( 10 men, 30 women) aged $\geq 80$ years who underwent BHA using the CPP approach. The average age of the subjects was 85.8 years (8094 years). We examined operation time, bleeding, preservation of short external rotator muscles, complications, and stem alignment and subsidence from postoperative radiographs.

\section{Results}

Although, gemellus inferior muscle injury was detected in 4 patients (10\%), hip joint stability was very excellent in all cases. There was no intraoperative fracture and postoperative dislocation. On postoperative radiographs, all femoral stems were in a neutral position. There was no stem subsidence in all the patients.

\section{Conclusions}

BHA using the CPP approach was not associated with postoperative dislocation and appeared to be useful even in geriatric patients with soft tissue fragility.

\section{Introduction}

The dislocation rate in conventional posterior approach in bipolar hemiarthroplasty $(\mathrm{BHA})$ is double that in the anterior approach [1, 2]. However, immobilization to reduce the risk of postoperative dislocation after BHA can delay rehabilitation [3]. Preserving soft tissue in BHA is important for reducing the risk of postoperative dislocation, and a variety of surgical approaches have been reported recently considering this complication [4]. The preservation technique of the piriformis tendon during the posterolateral approach in total hip arthroplasty (THA) is reportedly superior to the reattachment technique in terms of contiguity, muscle atrophy, and dislocation [5]. Kim et al. reported a method of THA that preserves muscles from the piriformis muscle to the internal obturator muscle (external rotator preservation procedure [ERP]) and had good outcomes with no cases of postoperative dislocation [6]. 
We performed BHA using a conjoined tendon-preserving posterior (CPP) approach, which only dissects the external obturator muscle and preserves the gemellus inferior muscle. However, extra care is needed with muscle- and tendon-preserving approaches in geriatric patients, due to the fragility of their soft tissue. To our knowledge, no previous study has reported whether fragile soft tissue can be preserved in geriatric patients using the CPP approach.

The objective of this study was to make the first report on BHA using the CPP approach in geriatric patients aged $\geq 80$ years and to examine their intraoperative findings and postoperative courses. We hypothesized that the CPP approach could be adaptable enough for geriatric patients with fragile soft tissue.

\section{Methods}

\section{Patient selection and study design}

This study was approved by the Ethics Committee of the institution. This was a retrospective review of 40 consecutive geriatric patients aged $\geq 80$ years who underwent BHA using CPP approach in our hospital from February 2017 to March 2018. This study included 10 men and 30 women. Mean age was 85.8 years (80-94 years). The mean height was $152 \mathrm{~cm}$ (range, $141-168 \mathrm{~cm}$ ); the mean body mass index was $19.3 \mathrm{~kg} / \mathrm{m}^{2}$ (range, $17.1-25.6 \mathrm{~kg} / \mathrm{m}^{2}$ ). The preoperative diagnoses were femoral neck fracture for all the patients. The Garden classification of the fracture type [7] was III for 12 hips (30\%) and IV for 28 hips (70\%). The femoral components used in this study were M/L Taper Hip Prosthesis with Kinectiv Technology (Zimmer Biomet G.K., Tokyo, Japan) for 32 patients, Alloclassic-SL ${ }^{\mathrm{T}}$ system (Zimmer Biomet G.K., Tokyo, Japan) for 6 patients, and PerFix HA femoral stem (Kyocera Medical, Kyoto, Japan) for 2 patients. M/L Taper Hip Prosthesis with Kinectiv Technology is a modular femoral stem system, and we can change femoral offset and leg length independently [8]. Alloclassic-SL ${ }^{\mathrm{TM}}$ system is a titanium alloy straight femoral stem with press-fit primary fixation with a forged titanium alloy Protasul- $100^{\mathrm{TM}}$ where niobium is replaced vanadium. PerFix HA femoral stem is a straight femoral stem coated with hydroxyapatite using a titanium arc-spray technique [9]. A $28 \mathrm{~mm}$-ceramic femoral head was used in all hips. The Dorr classification of the bone type [10] was A for 2 hips (5.0\%), B for 14 hips (35\%), and C for 24 hips (60\%). Mean postoperative follow-up period was 14 months (range, 12-24 months).

\section{Surgical procedure and postoperative care}

The operation is performed with the patients in the lateral decubitus position. In order to refer to a stable pelvis, a pelvic fixation device that has sacral and iliac pads is used. A 10-cm skin incision is made posteriorly at a $45^{\circ}$ angle from the posterior margin of the tip of the greater trochanter (Fig. 1a). The gluteus maximus is separated along its fibers to expose the posterior margin of the gluteus medius and the short external rotator muscles. The sciatic nerve is distinguished during this step, and the surgery is performed while confirming its position and protecting it. At this time, it is important to identify the external obturator muscle and trochanteric branch of the deep medial circumflex femoral artery. This 
artery should be cauterized at the proximal margin of the quadratus femoris muscle. Starting proximally, identify the piriformis, gemellus superior, internal obturator, gemellus inferior, and external obturator muscles (Fig. 1b). Incise the capsule posteriorly along the caudal margin of the gemellus inferior muscle. Extend the incision distally along the posterior border of the femur and invert the external obturator muscle and capsule (Fig. 1c). Thread the external obturator muscle and gemellus inferior muscle, respectively. Next, remove the femoral head after performing the osteotomy of the femoral neck. Performing osteotomy of the femoral neck beforehand makes it easier to remove the femoral head without muscle damage. Measure the femoral head and insert a trial cup into the acetabulum to determine the size of the outer head.

Perform femoral broaching while preserving and making sure not to damage the piriformis muscle and conjoined tendon covering the intertrochanteric notch, then determine the optimal stem size (Fig. 1d). Place an inner head and outer head of appropriate length to perform trial reduction. The most difficult part of this surgical technique is the manual reduction. Reduce the hip joint after flexing the hip joint until stem and conjoined tendon are parallel without excessive traction. In addition, perform reduction with the hip in mild abduction so there is no tension on the conjoined tendon. If the outer head can move to the acetabulum, reduction can be performed by pushing in the outer head. After confirming there is no leg length discrepancy, insert an implant of the same size (Fig. 1e, f). Repair the L-shaped incisions of the external obturator muscle and capsule, then close the wound after irrigation (Fig. 1g, h). All the patients began full weight-bearing exercises and range of motion exercises without restriction on postoperative day 1 . No brace, such as that with contraindicated limb positions or abduction pillows, was performed.

\section{Evaluations}

We examined operation time, bleeding, preservation of short external rotator muscles, complications, stem alignment [11], and subsidence from postoperative radiographs. Preservation of the short external rotator muscles was assessed by checking if any of the thread placed in the gemellus inferior muscle at the beginning of the operation remained when the wound was closed. Hip function was evaluated using the Harris Hip Score (HHS) [12] and Japanese Orthopaedic Association (JOA) hip score [13]. JOA hip score allocates 40 points for pain, 20 points for range of motion, 20 points for walking ability, and 20 points for activities of daily living, with a maximum total score of 100 points.

\section{Statistical analysis}

Preoperative and postoperative HHS and JOA hip scores were compared using a two-sample $t$-test. The differences were considered significant at a $p$ value $<0.05$. Statistical analysis was conducted using SPSS for Windows version 25 (IBM Corporation, Armonk, NY, USA).

\section{Results}

The operation time was 58 minutes (range, 40-75 minutes) and intraoperative bleeding was $150 \mathrm{ml}$ (range, $50-350 \mathrm{ml}$ ). There were 4 patients (10\%) whose gemellus inferior muscle was damaged. There were 2 patients (5\%) whose gemellus inferior muscle was difficult to identify. Mean stem subsidence was 
$0.2 \mathrm{~mm}$ (range, 0-1.6 mm), however, none showed significant subsidence of $\geq 3 \mathrm{~mm}$. On postoperative radiographs, all the femoral stems $(100 \%)$ were in a neutral position. The mean HHS and JOA hip scores were 83.4 points (range, 71-97 points) and 71.3 points (range, 55-88 points) preoperatively, and 82.5 points (range, 67-96 points) and 73.6 points (range, 58-92 points) at the final follow-up, respectively. There was no significant difference between the HHSs and JOA hip scores of before and that after surgery ( $p=0.24,0.30$, respectively). There were no cases of fracture, infection, or dislocation intraoperatively or postoperatively, and none of the hips required revision. Postoperative deep venous thrombosis was confirmed in 4 patients $(10 \%)$.

\section{Discussion}

We performed the BHA using a novel method, the CPP approach, in geriatric patients aged $\geq 80$ years. Making the capsule incision between the gemellus inferior and external obturator muscles allowed us to preserve the conjoined tendons except for that of the external obturator muscle, in almost all the patients.

The aging of society has led to more opportunities for performing BHA in geriatric patients. It can be difficult to instruct these patients on how to prevent dislocations due to their lack of understanding of limb positions that may cause dislocation. As hospital stays need to be shortened to reduce medical costs, there is a need for surgical methods that prevent dislocation because of daily activities, excluding falls and other accidents.

The most common conventional posterior approach involves incising the capsule and short external rotator muscles separately. Recently, methods have been employed that incise the capsule and short external rotator muscles together instead of separately, or only preserving the piriformis muscle. The short external rotator muscles and capsule play an important role in preventing hip dislocation. The short external rotator muscles and ischiofemoral ligament provide dynamic stability by controlling internal rotation, and the capsule provides static stability by acting as a posterior wall, both of which prevent dislocation. However, once the capsule and short external rotator muscles are cut, they have been found to re-rupture postoperatively at high rates of $75-92 \%$, even after being repaired $[14,15]$. Han et al. performed BHA using ERP in femoral neck fracture patients with mental disorder [4]. They reported that compared with the conventional posterior approach, there were no differences in operation time and bleeding, however, the rate of postoperative dislocation was significantly lower.

The CPP approach preserves from the piriformis muscle to the gemellus inferior muscle. Because this approach preserves the gemellus inferior muscle, we consider that this approach is superior to ERP at preventing postoperative dislocation. Moreover, the subjects in the present study were all geriatric patients aged $\geq 80$ years who may have had soft tissue fragility. In this study, the gemellus inferior muscle was difficult to identify in 2 cases and muscle-tendon damage was observed in 4 cases. The Alloclassic-SL ${ }^{\mathrm{TM}}$ system was used in the 4 patients with gemellus inferior muscle damage. These muscles are thought to have been damaged by the shoulder of the stem during rasping. Therefore, when using this approach, it is recommended to use a taper wedge type stem with reduced stem shoulder. That being 
said, due to individual differences in the shape of the conjoined tendon and external obturator muscle insertion sites, some degree of gemellus inferior muscle damage during rasping may be unavoidable. However, hip joint stability was very excellent in all cases and there were no cases of postoperative dislocation. The 4 cases with gemellus inferior muscle damage were highly resistant to posterior dislocation owing to the intact posterior capsule and ischiofemoral ligament, which indicates the CPP approach is useful in geriatric patients. The Sparing Piriformis muscle and Internus, Repair Externus (SPAIRE) technique is a muscle sparing mini-posterior approach for the THA reported by Handy et al. [16]. This approach enables preservation of the piriformis muscle tendon and conjoined insertion of obturator internus and the gemelli. However, a posterior capsulotomy is performed in an L-shape with a proximal oblique limb (at 10 o'clock for right hip, 2 o'clock for left hip) starting subjacent to the preserved quadriceps coxae tendons [16]. Therefore, this approach has 2 shortcomings. The first is the lower resistance to posterior dislocation from the posterior capsule than the CPP approach. The second is that due to the lack of a posterior capsule and the exposed conjoined tendon, the gemellus inferior muscle is more likely to be damaged than with the CPP approach.

Many young and inexperienced surgeons perform BHA for femoral neck fractures. Although the posterior approach is technically simple and provides a good field of view, we believe that the BHA with the CPP approach would be easy to adopt for surgeons with some experience with the posterior approach, because this approach needs no specialized equipment. While there is a surgical related learning curve, the operation time is less than 1 hour and if the surgical technique proves difficult the surgeon can switch to the conventional posterior approach. The CPP approach, which is highly resistant to posterior dislocation, can be considered a highly useful method for femoral neck fractures that accompany aging.

However, this study had a limitation and weaknesses. The sample size of the current study was relatively small to evaluate the outcomes of CPP approach. To validate our findings, future studies with larger sample sizes will be required.

In conclusion, we attempted to preserve the short external rotator muscles in BHA using the CPP approach in geriatric patients with soft tissue fragility. There were no intraoperative problems or postoperative dislocations, indicating that the CPP approach is useful for geriatric patients as well.

\section{Abbreviations}

BHA: bipolar hemiarthroplasty; THA: total hip arthroplasty; CPP: conjoined tendon-preserving posterior; HHS: Harris Hip Score; JOA: Japanese Orthopaedic Association; ERP: external rotator preservation; SPAIRE: sparing piriformis muscle and internus, repair externus

\section{Declarations}

\section{Acknowledgments}

We would like to thank Editage (www.editage.com) for English language editing. 


\section{Author contributions}

Conceptualization: Tomonori Tetsunaga, Toshifumi Ozaki.

Data curation: Tomonori Tetsunaga, Kazuki Yamada, Tomoaki Sanki, and Yoshi Kawamura.

Formal analysis: Tomonori Tetsunaga.

Investigation: Tomonori Tetsunaga.

Methodology: Tomonori Tetsunaga, Kazuki Yamada, Tomoaki Sanki, Yoshi Kawamura, and Toshifumi Ozaki.

Supervision: Tomonori Tetsunaga, Tomoko Tetsunaga, and Toshifumi Ozaki.

Writing-original draft: Tomonori Tetsunaga.

Writing-review and editing: Tomonori Tetsunaga, Tomoko Tetsunaga, and Toshifumi Ozaki.

\section{Funding}

No funding was received by any of the authors for this study.

\section{Availability of data and materials}

All data used and analyzed during this study are available from the corresponding author upon reasonable request.

\section{Ethics approval and consent to participate}

The Okayama University Hospital ethics committee approved this research.

\section{Consent for publication}

Not applicable.

\section{Competing interests}

The authors declare that they have no competing interests.

\section{References}

1. Kim Y, Kim JK, Joo IH, Hwang KT, Kim YH. Risk Factors Associated with Dislocation after Bipolar Hemiarthroplasty in Elderly Patients with Femoral Neck Fracture. Hip Pelvis. 2016;28(2):104-11.

2. Varley J, Parker MJ. Stability of hip hemiarthroplasties. Int Orthop. 2004;28(5):274-7. 
3. Muraki S, Yamamoto S, Ishibashi H, Nakamura K. Factors associated with mortality following hip fracture in Japan. J Bone Miner Metab. 2006;24(2):100-4.

4. Han SK, Kim YS, Kang SH. Treatment of femoral neck fractures with bipolar hemiarthroplasty using a modified minimally invasive posterior approach in patients with neurological disorders. Orthopedics. 2012;35(5):e635-40.

5. Minokawa S, Naito M, Shiramizu K, Nakamura Y, Kinoshita K, Minamikawa T, et al. Preservation technique of the piriformis tendon is superior to reattachment technique in terms of contiguity and muscle atrophy: using magnetic resonance imaging. Hip Int. 2018;28(6):599-605.

6. Kim YS, Kwon SY, Sun DH, Han SK, Maloney WJ. Modified posterior approach to total hip arthroplasty to enhance joint stability. Clin Orthop Relat Res. 2008;466(2):294-9.

7. Garden RS. Low-angle fixation in fractures of the femoral neck. J Bone Joint Surg. 1961;43-B:64763.

8. Shoji T, Yamasaki T, Izumi S, Hachisuka S, Ochi M. The influence of stem offset and neck shaft angles on the range of motion in total hip arthroplasty. Int Orthop. 2016;40(2):245-53.

9. Nakashima Y, Sato T, Yamamoto T, Motomura G, Ohishi M, Hamai S, et al. Results at a minimum of 10 years of follow-up for AMS and PerFix HA-coated cementless total hip arthroplasty: impact of cross-linked polyethylene on implant longevity. J Orthop Sci. 2013;18(6):962-8.

10. Dorr LD, Faugere MC, Mackel AM, Gruen TA, Bognar B, Malluche HH. Structural and cellular assessment of bone quality of proximal femur. Bone. 1993;14(3):231-42.

11. Lecerf G, Fessy MH, Philippot R, Massin P, Giraud F, Flecher X, et al. Femoral offset: anatomical concept, definition, assessment, implications for preoperative templating and hip arthroplasty. Orthop Traumatol Surg Res. 2009;95(3):210-9.

12. McGrory BJ, Harris WH. Can the western Ontario and McMaster Universities (WOMAC) osteoarthritis index be used to evaluate different hip joints in the same patient? J Arthroplasty. 1996;11(7):841-4.

13. Hasegawa Y, Iwata H, Mizuno M, Genda E, Sato S, Miura T. The natural course of osteoarthritis of the hip due to subluxation or acetabular dysplasia. Arch Orthop Trauma Surg. 1992;111(4):187-91.

14. Stahelin T, Drittenbass L, Hersche O, Miehlke W, Munzinger U. Failure of capsular enhanced short external rotator repair after total hip replacement. Clin Orthop Relat Res. 2004(420):199-204.

15. Loiba V, Stucinskas J, Robertsson O, Wingstrand H, Tarasevicius S. The analysis of posterior soft tissue repair durability after total hip arthroplasty in primary osteoarthritis patients. Hip Int. 2015;25(5):420-3.

16. Hanly RJ, Sokolowski S, Timperley AJ. The SPAIRE technique allows sparing of the piriformis and obturator internus in a modified posterior approach to the hip. Hip Int. 2017;27(2):205-9.

\section{Figures}


a

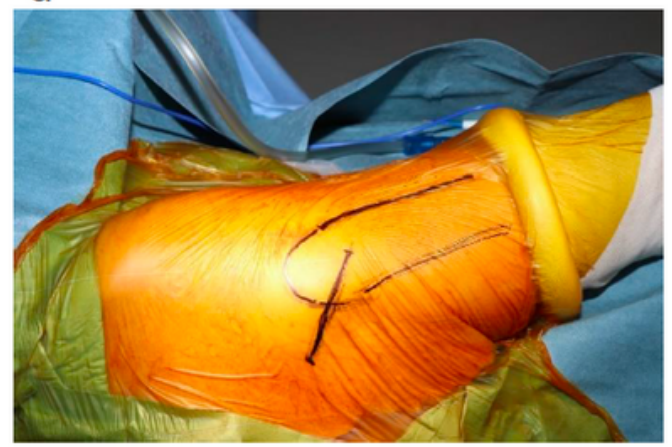

C

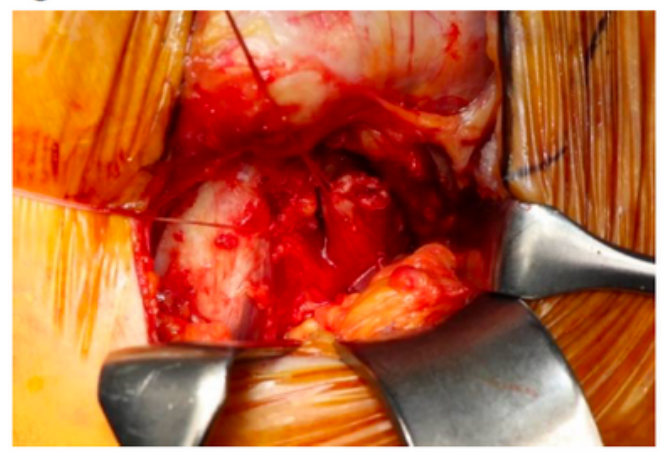

e

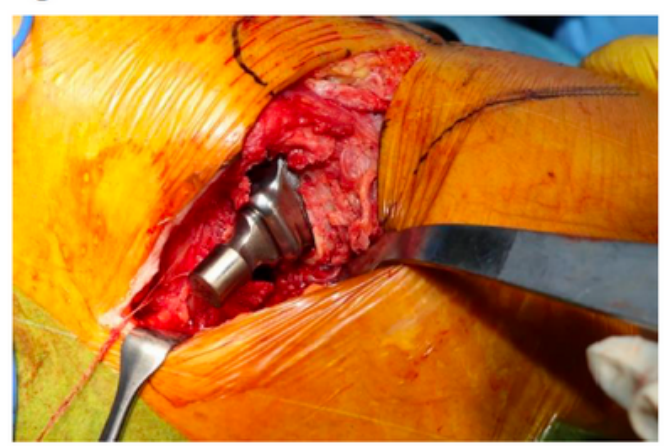

g

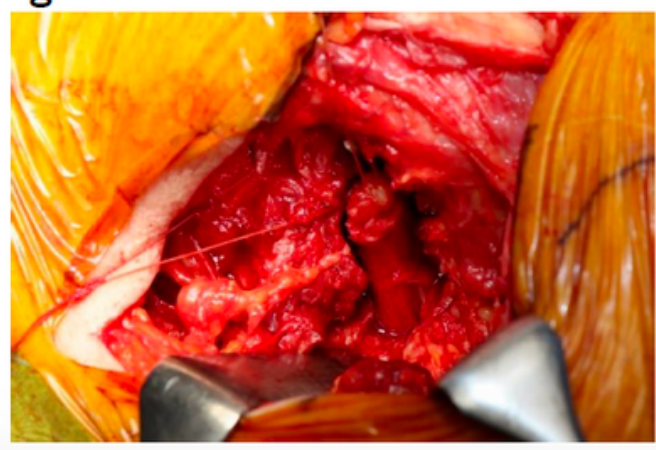

b

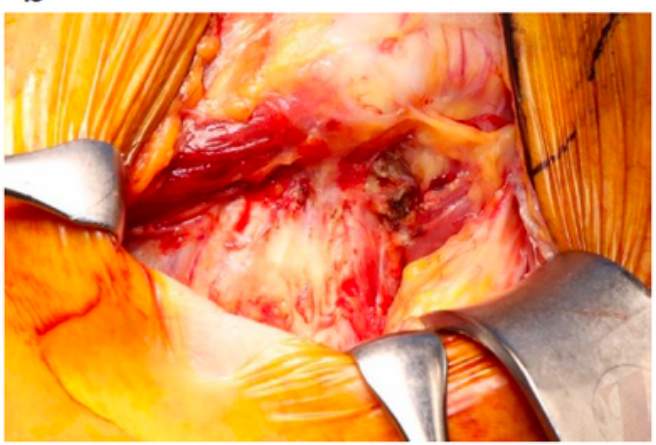

d

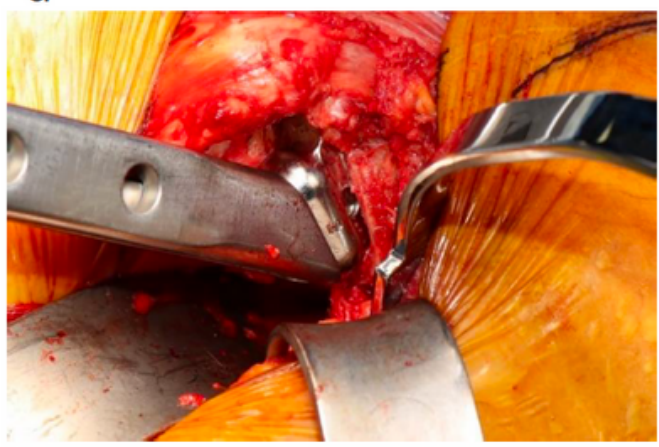

f

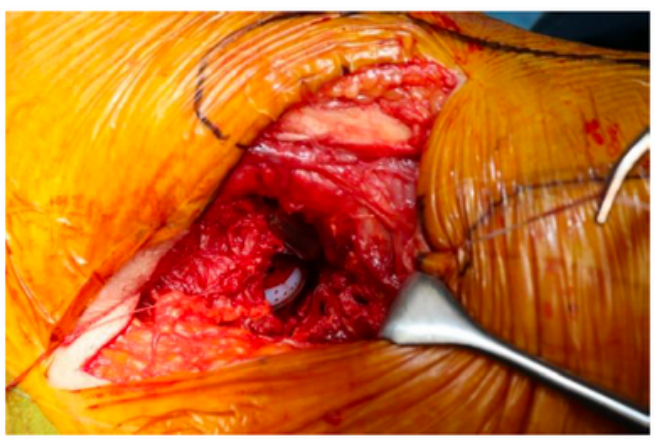

$\mathrm{h}$

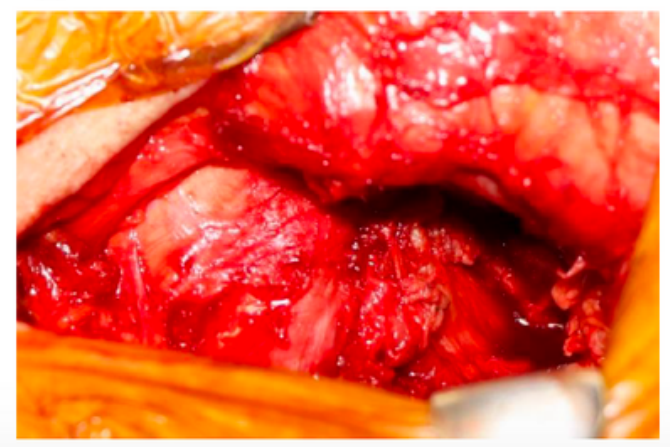

\section{Figure 1}

Skin incision (a). Short external rotator muscles (b). L-shaped incision in external obturator muscle and capsule (c). Femoral broaching (d). Stem insertion (e). After implant reduction (f). Before (g) and after (h) repair of the external obturator muscle and capsule. 